\title{
Factors associated with the presence of parafoveal scotoma in glaucomatous eyes with optic disc hemorrhages
}

\author{
Diego Torres Dias ${ }^{1,2}$ - Izabela Almeida ${ }^{1,2}$ - Adriana Miyuki Sassaki ${ }^{1} \cdot$ Verena Ribeiro Juncal $^{1} \cdot$ Michele Ushida $^{2}$. \\ Flavio Siqueira Lopes ${ }^{1,2} \cdot$ Paula Alhadeff ${ }^{3}$. Robert Ritch $\mathbb{1}^{3} \cdot$ Tiago Santos Prata $\mathbb{( 1 )}^{1,2,4}$
}

Received: 15 April 2017 / Revised: 21 May 2018 / Accepted: 29 May 2018 / Published online: 4 July 2018

(c) The Royal College of Ophthalmologists 2018

\begin{abstract}
Purpose Glaucomatous eyes with disc hemorrhage (DH) have a greater risk of paracentral visual field (VF) loss. However, not every DH eye presents with parafoveal scotoma (PFS), and contributing factors are still to be determined. In the present study, we investigated clinical and ocular factors associated with the presence of PFS in glaucomatous eyes with DH.

Methods A case-control study was carried out. One hundred thirty glaucomatous patients with DH were enrolled. They were divided into two groups based on two reliable 24-2 VF tests: those with PFS (defined as $\geq 3$ adjacent points with $p<5 \%$ within the central 10 degrees of fixation, $\geq 1$ point with $p<1 \%$ lying at the innermost paracentral points, in the same hemifield) and those without PFS. Clinical and ocular data from the time of DH detection were compared between groups. Factors associated with the presence of PFS were investigated through logistic regression.

Results The PFS group had a higher prevalence of Caucasian patients (82 vs. 47\%; $p<0.01$ ). Eyes with PFS had a more negative spherical equivalent and worse VF mean deviation (MD) index $(p \leq 0.01)$. There was a marginally significant intraocular pressure (IOP) difference between eyes with $(15 \mathrm{mmHg})$ and without PFS $(18 \mathrm{mmHg})$ at the time of DH detection $(p=0.10)$. Univariable analysis revealed PFS to be significantly associated with Caucasian race (OR, 3.02; $p=$ $0.004)$, myopia (<-3 diopters; OR, 3.44; $p=0.039)$, and lower IOP $(\leq 16 \mathrm{mmHg}$; OR, $2.10 ; p \leq 0.047)$. Multivariable analysis, controlling for VF MD, revealed that only Caucasian race and myopia (as a continuous or categorical variable) remained significant in this model $(p \leq 0.038)$.

Conclusions Caucasian race and the presence and magnitude of myopia were found to be significantly associated with the presence of PFS in glaucomatous eyes with DH. Our results may help clinicians in the identification and surveillance of these eyes at higher risk of central VF loss.
\end{abstract}

\section{Introduction}

Glaucoma is a progressive disease, characterized by retinal ganglion cell degeneration, which results in optic nerve

Tiago Santos Prata

t.prata0807@gmail.com

1 Department of Ophthalmology, Federal University of São Paulo, São Paulo, Brazil

2 Glaucoma Unit, Hospital Medicina dos Olhos - HMO, Osasco, Brazil

3 Einhorn Clinical Research Center, New York Eye and Ear Infirmary of Mount Sinai, New York, NY, USA

4 Department of Ophthalmology, Glaucoma Service, Hospital Oftalmológico de Sorocaba - BOS, Sorocaba, Brazil head alterations and associated visual field (VF) loss [1]. Traditionally, glaucoma has been considered to spare central visual function until late in the disease process. However, many patients have VF defects in the paracentral region earlier in the disease process [2-5]. Parafoveal scotomas (PFS) are of great concern as they represent a higher risk of visual acuity loss [6], and are associated with greater disability in routine activities, such as reading [7] and driving[8].

The presence of optic disc hemorrhages (DH) is also a frequent concern for glaucoma specialists. DH is a wellestablished risk factor for glaucoma development [9] and disease progression [10-13]. Although described over 100 years ago $[14,15]$, its underlying causative mechanisms are still not well established. It is believed that intraocular pressure (IOP)-independent mechanisms, such as vascular dysregulation [16, 17] and lamina cribrosa (LC) 
biomechanical alterations [18-20] could play an important role in DH pathogenesis. Furthermore, when a patient presents with a DH, the clinical implications for that specific individual are highly variable [21], as glaucoma development [9] and VF progression [10-13] rates differ significantly between patients with $\mathrm{DH}$.

Some authors suggest that PFS may also develop in response to similar IOP-independent mechanisms. That hypothesis is supported by the higher detection frequency of PFS in patients with normal-tension glaucoma (NTG) [2, 22], a subgroup of patients, which is known to also have a higher frequency of DHs [12]. Within this context, recent reports have identified $\mathrm{DH}$ as a significant risk factor for the presence of PFS in glaucomatous eyes [23, 24]. However, not every DH eye presents with a PFS[23], and possible contributing factors are still to be determined in these cases. In the present study, we sought to assess ocular and clinical factors associated with the presence of PFS in glaucomatous eyes with DH.

\section{Materials and methods}

This protocol adhered to the tenets of the declaration of Helsinki and was approved by the ethics committee and the institutional review board of the New York Eye and Ear Infirmary and the Federal University of São Paulo.

\section{Participants}

In this case-control study, we enrolled consecutive glaucomatous patients with DH followed at the Glaucoma Services of the two above-mentioned institutions. On the basis of at least two previous reliable VFs, these DH patients were divided into two groups: those with PFS (case group) and those without PFS (control group). All data were collected cross-sectionally (at the time of DH detection). Clinical and ocular characteristics from the time of DH detection were compared between patients with and without PFS.

All participants had undergone a comprehensive ophthalmological evaluation, including best-corrected visual acuity, slit-lamp biomicroscopy, IOP measurement, gonioscopy, dilated fundoscopy, VF testing (24-2 Swedish interactive threshold algorithm, Humphrey Field Analyzer II; Carl Zeiss Meditec, Inc., Dublin, CA), optic disc stereophotographs, and color/red-free fundus imaging. Disc photographs of all patients were reviewed by two glaucoma specialists for the presence of DH. In cases of disagreement, the impression of a third investigator was used for adjudication. A DH was defined as a splinterlike or flame-shaped hemorrhage on or within the retinal nerve fiber layer (RNFL) or neuroretinal rim. If peripheral to the disc margin, the hemorrhage needed to be contiguous to the $\beta$-zone parapapillary atrophy (PPA) when this feature was present [25]. Exclusion criteria were the presence of diabetic retinopathy, vascular occlusive disease, optic disc drusen, ocular trauma, and recent history of posterior vitreous detachment.

Glaucomatous optic neuropathy was defined as cup-todisc ratio $>0.6$, asymmetry between eyes $\geq 0.2$, presence of localized defects of the RNFL, and/or neuroretinal rim in the absence of any other anomalies that could explain such findings. Characteristic glaucomatous VF defect was defined as glaucoma hemifield test results outside normal limits and the presence of at least three contiguous test points within the same hemifield on the pattern deviation plot at $p<1 \%$, with at least one at $p<0.5 \%$, excluding points on the edge of the field or those directly above and below the blind spot.

\section{Data collection, VF, and optic disc assessment}

The timepoint for purposes of data acquisition was defined as the date at which the DH was documented photographically. Clinical and ocular data included age, race, gender, IOP, central corneal thickness (CCT), refractive error, VF mean deviation (MD), presence of PFS, optic disc phenotype, presence of PPA, and RNFL defects; types of previous intraocular surgeries (such as phacoemulsification, trabeculectomy, and combined procedures); type of glaucoma, DH location and recurrence. Patient-related history of systemic vascular diseases (systemic hypertension or diabetes) and signs/symptoms of primary vascular dysregulation (migraine, Raynaud's phenomenon, cold hands, and low blood pressure) were also documented.

The classifications of optic disc phenotype [26] and the presence of PFS [23] were based on previously published studies. Briefly, discs were classified into four subtypes: focal loss (type 1), myopic (type 2), senile sclerotic (type 3 ), and generalized cup enlargement (type 4) [26]. PFS was defined as a VF defect in one hemifield within $10^{\circ}$ of fixation with at least one point at $p<1 \%$ lying at the two innermost paracentral points, with or without defects outside the central $10^{\circ}$ in the superior or inferior arcuate area [23]. Whenever both eyes were eligible, one was randomly selected for analysis.

\section{Statistical analysis}

Descriptive analysis was used to present the demographic and clinical data. Data with normal distribution were analyzed with independent samples $t$-test, and those nonnormally distributed by the Mann-Whitney test. Categorical data were analyzed with the chi-square test. Multiple logistic regression analysis was used to evaluate patients' characteristics associated with the presence of PFS. First, 
Table 1 Comparison of the clinical and demographic characteristics between patients with and without parafoveal scotoma

\begin{tabular}{llll}
\hline & $\begin{array}{l}\text { PFS group } \\
(n=77)\end{array}$ & $\begin{array}{l}\text { Non-PFS group } \\
(n=53)\end{array}$ & $p$ Value \\
\hline Age (years) & $68.2 \pm 10.9$ & $65.1 \pm 10.8$ & 0.12 \\
Gender (\%; F/M) & $66 / 34$ & $69 / 31$ & 0.81 \\
Race (\%; Caucasian/Asian/Others) & $82 / 9 / 9$ & $47 / 26 / 27$ & $<0.01$ \\
Systemic vascular disease (DM or & 59 & 63 & 0.77 \\
hypertension) & & & 0.29 \\
Features of systemic vascular & 18 & 10 & \\
dysregulation (\%) & & & $<0.01$ \\
Spherical equivalent (diopters) & $-1.4 \pm 2.9$ (range: -11.50 to & $0.1 \pm 2.6$ (range: \\
& $+3.25)$ & -10 to +5$)$ & $<0.01$ \\
MD index (dB) & $-7.8 \pm 7.1$ & $-4.2 \pm 4.4$ & 0.10 \\
Mean IOP at DH detection (mmHg) & 15 & 18 & 0.86 \\
Central corneal thickness ( $\mu$ m) & $530.7 \pm 40.7$ & $531.5 \pm 47.7$ & 0.68 \\
NTG diagnosis (\%) & 33 & 28 & 0.47 \\
Previous intraocular surgery (\%) & 29 & 23 & 0.48 \\
Optic disc phenotypes (\%; types 1/2/3/4) & $48 / 3 / 18 / 31$ & $46 / 12 / 11 / 31$ & 0.48 \\
Presence of PPA (\%) & 91 & 80 & 0.37 \\
Localized RNFL defect $(\%)$ & 76 & 65 & 0.35 \\
DH location (\%; inferior temporal/ & $73 / 27$ & $84 / 16$ & 0.25 \\
others) & & 28 & \\
DH recurrences (\%) & 18 & &
\end{tabular}

Data given as mean \pm standard deviation whenever indicated

$P F S$ parafoveal scotoma, $M$ male, $F$ female, $D M$ diabetes mellitus, $M D$ mean deviation, $I O P$ intraocular pressure, $D H$ disc hemorrhage, $N T G$ normal-tension glaucoma, $P P A$ peripapillary atrophy, $R N F L$ retinal nerve fiber layer

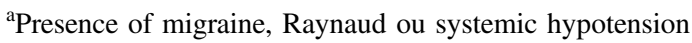

each variable was analyzed in a univariable model. Then, all variables with a significance level of less than 0.10 were included in the multivariable model [27]. Statistical significance was set at $p<0.05$. The role of each variable was expressed in odds ratio (OR) and its 95\% confidence intervals. Computerized analysis was performed using MedCalc software (MedCalc, Inc., Mariakerke, Belgium).

\section{Results}

A total of 130 consecutive patients with $\mathrm{DH}$ were enrolled (72 from the New York Center and 58 from the Brazilian Center). The division according to the presence of PFS at the time of DH detection resulted in 77 eyes with PFS and 53 eyes without PFS (Table 1). The PFS group had a higher prevalence of Caucasian patients ( 82 vs. $47 \%$; $p<0.01$ ), a more negative spherical equivalent and a worse VF MD index $(p \leq 0.01)$. Although eyes with PFS had lower IOP values than eyes without PFS at the time of DH detection (median, $15 \mathrm{mmHg}$ vs. $18 \mathrm{mmHg}$ ), this difference did not reach statistical significance $(p=0.10)$. When it comes to ethnic characteristics, the majority of the Asian patients were recruited from the Brazilian Center. This Asian population was uniformly composed by Japanese descendants.

No significant differences were found regarding age, gender, CCT, optic disc phenotype, presence of PPA, localized RNFL defects, numbers of previous intraocular surgery, type of glaucoma, systemic risk factors, DH location, and DH recurrences $(p \geq 0.12)$.

Regarding the investigation of factors possibly associated with the presence of PFS in these glaucomatous eyes with DH, logistic regression analysis revealed PFS to be significantly associated with Caucasian race (OR, 3.02; $p=$ 0.004 ), myopia ( $<-3 \mathrm{D}$; OR, 3.44; $p=0.039$ ), and lower IOP $(\leq 16 \mathrm{mmHg}$; OR, $2.10 ; p \leq 0.047)$. Multivariable analysis, including all significant variables (and controlling for VF MD index), revealed that only Caucasian race and myopia (as a continuous or categorical variable) remained significant in this model $(p \leq 0.038)$. Multivariable analysis data are provided in detail in Table 2.

\section{Discussion}

The presence of PFS and DH is frequent concern for the glaucoma specialist. It is well established that DHs are 
Table 2 Factors associated with the presence of parafoveal scotoma in glaucomatous eyes with disc hemorrhage

\begin{tabular}{|c|c|c|c|c|c|c|}
\hline & \multicolumn{3}{|c|}{ Univariable analysis } & \multicolumn{3}{|c|}{ Multivariable analysis ${ }^{\text {a.b }}$} \\
\hline & $\begin{array}{l}\text { Odds } \\
\text { ratio }\end{array}$ & $\begin{array}{l}95 \% \text { confidence } \\
\text { interval }\end{array}$ & $p$ & $\begin{array}{l}\text { Odds } \\
\text { ratio }\end{array}$ & $\begin{array}{l}95 \% \text { confidence } \\
\text { interval }\end{array}$ & $p$ \\
\hline Age (years) & 1.03 & $0.99-1.06$ & 0.113 & & & \\
\hline Race (Caucasian/Others) & 3.02 & $1.43-6.38$ & 0.004 & 3.42 & $1.47-7.96$ & 0.004 \\
\hline $\begin{array}{l}\text { Spherical equivalent } \\
\text { (diopters) }\end{array}$ & 0.83 & $0.71-0.97$ & 0.018 & 0.85 & $0.73-0.98$ & 0.032 \\
\hline Myopia (<-3D) & 3.44 & $1.06-11.15$ & 0.039 & 3.73 & $1.07-13.01$ & 0.038 \\
\hline $\begin{array}{l}\text { IOP at DH detection } \\
(\mathrm{mmHg})\end{array}$ & 0.94 & $0.87-1.02$ & 0.161 & & & \\
\hline $\begin{array}{l}\text { Low IOP at DH detection } \\
(\leq 16 \mathrm{mmHg})\end{array}$ & 2.10 & $1.01-4.36$ & 0.047 & 1.78 & $0.75-4.23$ & 0.188 \\
\hline
\end{tabular}

IOP intraocular pressure, $\mathrm{DH}$ disc hemorrhage

${ }^{a}$ Only variables with a $p$ value of less than 0.10 in the univariable analysis were included in the multivariable model

${ }^{\mathrm{b}}$ The variables were controlled for visual field mean deviation index associated with glaucoma development [9] and progression [10-13]; and PFS indicate a greater risk of visual acuity loss [6], driving issues [7], and reading disability [8]. With that in mind, clinicians are prompted to establish more rigid therapeutic regimens in these situations. Previous studies reported an association between DH occurrence and the presence of PFS in glaucomatous eyes [23, 24]. However, not every eye with a DH presents with a PFS. Investigating clinical and ocular features possibly associated with the presence of PFS in glaucomatous eyes with DHs, we found Caucasian race and the presence and magnitude of myopia to be significantly associated with paracentral VF presence in these eyes. The authors are unaware of other studies reporting such findings.

There are scant data in the literature regarding clinical parameters associated with PFS in glaucomatous eyes. Unfortunately, none of these studies focused specifically in glaucomatous eyes with DH, which partially limits a direct comparison with our study. Analyzing those published studies in glaucomatous eyes in general, myopic patients seem to be more prone to present with PFS than those without myopia [28-30]. Considering other factors, Park and colleagues reported the association between PFS and lower untreated IOP, DH, and systemic risk factors (hypotension, Raynaud's phenomenon, migraine, and sleep apnea) [23]. Rao and associates reported a higher frequency of DHs and increased vertical displacement of the central vessel trunk to be associated with PFS in glaucomatous eyes [24]. When evaluating a Caucasian population, Wiggs and colleagues, found that a specific genomic pattern (the p53 codon $72 \mathrm{PRO} / \mathrm{PRO}$ genotype) may be associated with early central VF loss in patients with primary open-angle glaucoma [31]. Since we found Caucasian patients to be more prone to presenting PFS than other races, we believe that genetic predisposition to PFS may play a role in this association, and our results corroborate Wiggs' findings. In our study, corroborating Park's results [23], we also found lower (treated) IOP to be associated with PFS. However, this association lost its significance when other variables were considered (multivariable analysis), being Caucasian race and the presence of myopia and its magnitude the only variables independently associated with paracentral VF presence in DH eyes. We believe the difference in our results can be explained in part by the fact that we evaluated a more specific population (only eyes with DH were included). With that in mind, we do not consider these results contradictory; in fact, we believe that they complement each other, as we add this new information about this subgroup of high-risk eyes.

In the face of our results, we believe it is important to delve into the close relationship between myopia and glaucoma. Not only is myopia significantly associated with glaucoma in general [32], but clinical studies have also suggested that myopia is a risk factor for PFS [28-30], for glaucoma progression in the central VF [33] and for glaucoma progression with IOP within the low-teens [33]. Park and colleagues proposed that glaucomatous patients could be divided according to their initial VF loss [23]. They suggest that, in patients who present with initial paracentral VF loss, glaucoma may be more associated with IOPindependent risk factors [23]. Our results corroborate in part these previous reports supporting the hypothesis that glaucoma in myopic patients is associated with earlier paracentral VF presence and possibly IOP-independent mechanisms. Explanations for this "myopic NTG" with earlier paracentral VF presence could be related to a greater anatomical susceptibility of these eyes, as a result of an increased axial length and greater deformability of the LC [34]. Histologic studies in myopic eyes have shown LC thinning and stretching, which may alter the trans-laminar 
pressure gradient due to a smaller distance between the intraocular (IOP) and the retrobulbar compartments (cerebrospinal fluid pressure) [35]. This increased exposure of the peripheral posterior LC surface, no longer buffered by solid optic nerve tissue, may lead to greater backward bowing of the LC and higher vulnerability to neuronal damage at lower IOP levels [35]. Enhanced-depth imaging and swept-source optical coherence tomography technologies are currently being applied for the investigation of LC contribution to glaucoma damage in vivo [36]; and the association between LC thinning [37] and LC defects [38] with glaucoma in myopic patients has also been reported, reinforcing the role of LC alterations in IOP-independent glaucoma mechanisms. Kim and colleagues also reported that $\mathrm{DH}$ in myopic eyes were more frequently located with its proximal location within the LC (compared to cup margin, disc rim, or peripapillary region), suggesting that the LC of a myopic disc has a higher vulnerability to glaucomatous damage as the proximal location of a $\mathrm{DH}$ represents the most stressed location in the optic nerve head [39]. Finalizing, previous studies also reported that myopic patients are more prone to presenting RNFL defects closer to the papillomacular bundle [28-30] than non-myopic patients, which could lead to earlier paracentral VF presence in these eyes.

We believe it is important to briefly discuss the main clinical implications of our findings. The main outcome of our study was the association between Caucasian race and myopia (and its magnitude) and the presence of PFS in DH eyes. Our findings indicated that Caucasian and myopic patients have a higher risk of presenting PFS at the time of DH detection than non-myopic patients. It has been well established that DH itself not only represents a higher risk for glaucoma development [9] and progression [10-13], but also for paracentral VF defects [23, 24]. On another hand, myopia itself is associated with a higher prevalence of PFS in glaucomatous eyes [28-30]. Based on our findings, one could infer that myopia remains a significant risk factor for PFS, even in these eyes that apparently already have a greater risk for PFS due to DH. Even though our data are not longitudinal and therefore does not allow the investigation of cause-effect relationships; we suggest that among these high-risk eyes (DH eyes) those with myopia deserve a closer monitoring of the paracentral VF. The higher the degree of myopia, more aggressive surveillance must be adopted by the clinician, as these patients must be closely monitored for VF progression within the paracentral area (which is closely related to quality of life) [6-8]. It might be worth considering VF assessment at shorter intervals and earlier central VF assessment with the 10-2 strategy during follow-up. It should be noted that some of these myopic patients may present PFS due to myopic maculopathy (more frequently observed in highly myopic eyes). It is important to highlight that these eyes were not included in our study.

The present study has some specific limitations that should be addressed. First, the prevalence of systemic risk factors was assessed on the basis of questionnaire data, which might have reliability and accuracy issues. Second, due to the nature of our data, we can establish possible associations, but not cause-effect relationships. This fact should be considered while interpreting our results. Third, although refractive error data were collected from all patients, we could only include those from phakic eyes in the analysis. Axial length data, which were not collected, would have been an important variable due to its relationship with axial myopia. Finally, even though we have adopted strict criteria to define a glaucomatous DH in our study, some patients with DHs related to other (non-glaucomatous) conditions might have been included.

In conclusion, in this large series of glaucomatous eyes with DHs, Caucasian race and the presence and magnitude of myopia (as a continuous or categorical variable) were found to be significantly associated with paracentral VF presence in DH eyes. Our results may help clinicians in the identification and surveillance of these eyes at higher risk of central field loss. Future longitudinal studies are warranted in order to confirm these initial findings based on crosssectional data.

\section{Summary}

\section{What was known before?}

- Eyes with disc hemorraghes and parafoveal scotomas are at higher risk of visual acuity loss.

\section{What this study adds?}

- Caucasian race and the presence and magnitude of myopia were found to be significantly associated with paracentral VF involvement in eyes with disc hemorrhages.

\section{Compliance with ethical standards}

Conflict of interest The authors declare that they have no conflict of interest.

\section{References}

1. Weinreb RN, Khaw PT. Primary open-angle glaucoma. Lancet. 2004;363:1711-20.

2. Hitchings RA, Anderton SA. A comparative study of visual field defects seen in patients with low-tension glaucoma and chronic simple glaucoma. Br J Ophthalmol. 1983;67:818-21. 
3. Caprioli J, Spaeth GL. Comparison of visual field defects in the low-tension glaucomas with those in the high-tension glaucomas. Am J Ophthalmol. 1984;97:730-7.

4. Motolko M, Drance SM, Douglas GR. Visual field defects in lowtension glaucoma: comparison of defects in low-tension glaucoma and chronic open angle glaucoma. Arch Ophthalmol. 1982;100: 1074-7.

5. King D, Drance SM, Douglas G, Schulzer M, Wijsman K. Comparison of visual field defects in normal-tension glaucoma and high-tension glaucoma. Am J Ophthalmol. 1986;101: 204-7.

6. Kolker AE. Visual prognosis in advanced glaucoma: a comparison of medical and surgical therapy for retention of vision in 101 eyes with advanced glaucoma. Trans Am Ophthalmol Soc. 1977;75:539-55.

7. Fujita K, Yasuda N, Oda K, Yuzawa M. Reading performance in patients with central visual field disturbance due to glaucoma. Nippon Ganka Gakkai Zasshi. 2006;110:914-8.

8. Coeckelbergh TR, Brouwer WH, Cornelissen FW, Van Wolffelaar P, Kooijman AC. The effect of visual field defects on driving performance: a driving simulator study. Arch Ophthalmol. 2002;120:1509-16.

9. Budenz DL, Anderson DR, Feuer WJ, Beiser JA, Schiffman J, Parrish RK 2nd, et al. Detection and prognostic significance of optic disk hemorrhages during the ocular hypertension treatment study. Ophthalmology. 2006;113:2137-43.

10. Prata TS, De Moraes CGV, Teng CC, Tello C, Ritch R, Liebmann JM. Factors affecting rates of visual field progression in glaucoma patients with optic disc hemorrhage. Ophthalmology. 2010;117: 24-9.

11. Collaborative Normal-Tension Glaucoma Study Group. Comparison of glaucomatous progression between untreated patients with normal-tension glaucoma and patients with therapeutically reduced intraocular pressures. Am J Ophthalmol. 1998;126: $487-7$.

12. Drance S, Anderson DR, Schulzer M. Risk factors for progression of visual field abnormalities in normal-tension glaucoma. Am J Ophthalmol. 2001;131:699-708.

13. Ishida K, Yamamoto T, Sugiyama K, Kitazawa Y. Disk hemorrhage is a significantly negative prognostic factor in normaltension glaucoma. Am J Ophthalmol. 2000;129:707-14.

14. Bjerrum J. Om en Tilfojelse til den saedvanlige synsfelfundersogelse samt om synfeltet ved glaukom. Nord Ophthalmol Tidsskr. 1889;2:141-85.

15. Bjerrum J. Om glaukomets kliniske afgraensning. Nord Ophthalmol Tidsskr. 1892;5:129-38.

16. Grieshaber MC, Flammer J. Does the blood-brain barrier play a role in glaucoma? Surv Ophthalmol. 2007;52(Suppl 2): S115-21.

17. Flammer J, Konieczka K, Flammer AJ. The primary vascular dysregulation syndrome: implications for eye diseases. EPMA J. 2013;4:14.

18. Lee EJ, Kim TW, Kim M, Girard MJ, Mari JM, Weinreb RN. Recent structural alteration of the peripheral lamina cribrosa near the location of disc hemorrhage in glaucoma. Invest Ophthalmol Vis Sci. 2014;55:2805-15.

19. Lee EJ, Han JC, Kee C. A novel hypothesis for the pathogenesis of glaucomatous disc hemorrhage. Prog Retin Eye Res. 2017;60:20-43.

20. Kim YK, Jeoung JW, Park KH. Effect of focal lamina cribrosa defect on disc hemorrhage area in glaucoma. Invest Ophthalmol Vis Sci. 2016;57:899-907.
21. Dias DT, Ushida M, Prata TS. Do all disc hemorrhages have the same causative mechanism and same influence on glaucoma prognosis? Am J Ophthalmol. 2016;166:208-9.

22. Gardiner SK, Johnson CA, Cioffi GA. Evaluation of the structurefunction relationship in glaucoma. Invest Ophthalmol Vis Sci. 2005;46:3712-7.

23. Park SC, De Moraes CG, Teng CC, Tello C, Liebmann JM, Ritch R. Initial parafoveal versus peripheral scotomas in glaucoma: risk factors and visual field characteristics. Ophthalmology. 2011;118:1782-9.

24. Rao A, Mukherjee S. Anatomical attributes of the optic nerve head in eyes with parafoveal scotoma in normal tension glaucoma. PLoS ONE. 2014;9:e90554.

25. Airaksinen PJ, Mustonen E, Alanko HI. Optic disc hemorrhages: analysis of stereophotographs and clinical data of 112 patients. Arch Ophthalmol. 1981;99:1795-801.

26. Broadway DC, Nicolela MT, Drance SM. Optic disk appearances in primary open-angle glaucoma. Surv Ophthalmol. 1999;43 (Suppl 1):S223-43.

27. Peters TJ. Multifarious terminology: multivariable or multivariate? univariable or univariate? Paediatr Perinat Epidemiol. 2008;22:506.

28. Araie M, Arai M, Koseki N, Suzuki Y. Influence of myopic refraction on visual field defects in normal tension and primary open angle glaucoma. Jpn J Ophthalmol. 1995;39:60-4.

29. Mayama C, Suzuki Y, Araie M, Ishida K, Akira T, Yamamoto T, et al. Myopia and advanced-stage open-angle glaucoma. Ophthalmology. 2002;109:2072-7.

30. Kimura Y, Hangai M, Morooka S, Takayama K, Nakano N, Nukada $M$, et al. Retinal nerve fiber layer defects in highly myopic eyes with early glaucoma. Invest Ophthalmol Vis Sci. 2012;53(10):6472-8.

31. Wiggs JL, Hewitt AW, Fan BJ, Wang DY, Figueiredo Sena DR, O'Brien $\mathrm{C}$, et al. The p53 codon 72 PRO/PRO genotype may be associated with initial central visual field defects in caucasians with primary open angle glaucoma. PLoS One. 2012;7:e45613 https://doi.org/10.1371/journal.pone.0045613.Epub 2012 Sep 26

32. Marcus MW, de Vries MM, Junoy Montolio FG, Jansonius NM. Myopia as a risk factor for open-angle glaucoma: a systematic review and meta-analysis. Ophthalmology. 2011;118:1989-94.

33. Sakata R, Aihara M, Murata H, Mayama C, Tomidokoro A, Iwase A, et al. Contributing factors for progression of visual field loss in normal-tension glaucoma patients with medical treatment. J Glaucoma. 2013;22:250-4.

34. Fong DS, Epstein DL, Allingham RR. Glaucoma and myopia: are they related? Int Ophthalmol Clin. 1990;30:215-8.

35. Jonas JB, Xu L. Histological changes of high axial myopia. Eye (Lond). 2014;28:113-7.

36. Park HY, Shin HY, Park CK. Imaging the posterior segment of the eye using swept-source optical coherence tomography in myopic glaucoma eyes: comparison with enhanced-depth imaging. Am J Ophthalmol. 2014;157:550-7.

37. Lopilly Park HY, Lee NY, Choi JA, Park CK. Measurement of scleral thickness using swept-source optical coherence tomography in patients with open-angle glaucoma and myopia. Am J Ophthalmol. 2014;157:876-84.

38. Han JC, Cho SH, Sohn DY, Kee C. The characteristics of lamina cribrosa defects in myopic eyes with and without open-angle glaucoma. Invest Ophthalmol Vis Sci. 2016;57:486-94.

39. Kim HS, Park KH, Jeoung JW, Park J. Comparison of myopic and nonmyopic disc hemorrhage in primary open-angle glaucoma. Jpn J Ophthalmol. 2013;57(2):166-71. 